\title{
Between sealing and leakiness: molecular dynamics of the endothelium to maintain and regulate barrier function
}

\author{
Hans Schnittler
}

Received: 30 January 2014 / Accepted: 30 January 2014 / Published online: 11 March 2014

(C) Springer-Verlag Berlin Heidelberg 2014

The vascular endothelium not only takes a strategically important anatomical position in our body by covering the inner lining of all lymphatic and blood vessels and the heart, thereby acting as a highly widespread "organ" along the $100,000 \mathrm{~km}$ of everyone's vascular tree but is also dynamically engaged in the regulation of blood pressure, hemostasis, inflammation and defense and in organ-specific perfusion. Hence, the endothelium functions as a selective barrier that tightly balances the exchange of water, solutes, macromolecules and mobile cells between the flowing blood and the underlying tissues. This balance is essential to achieve systemic and site-specific vascular homeostasis by responding to a wide variety of physiological and pathological stimuli, resulting in changes of intercellular junctions or trans-cellular transport processes. Thus, apart from their biological relevance in maintaining and regulating barrier function, endothelial cell junctions are major targets in many vascular diseases and in cell regeneration and repair, as they affect shape changes, barrier breakdown/ apoptosis or cell migration, and proliferation. In the last decade, a great deal of experimental work has been performed by many renowned laboratories to unravel the molecular dynamics of endothelial barrier function and to translate this knowledge into biomedical applications. In this special themed issue of Cell and Tissue Research, a collection of review articles will address the various mechanistic aspects of endothelial barrier function.

In their overview, entitled "Experimental tools to monitor the dynamics of endothelial barrier function: a survey of in vitro approaches", Wegener and Seebach (2014) introduce methods for experimentally studying endothelial barrier

H. Schnittler $(\bowtie)$

Institute of Anatomy \& Vascular Biology, WWU-Münster,

Vesaliusweg 2-4, Münster 48149, Germany

e-mail: hans.schnittler@uni-muenster.de function, e.g., by impedance spectroscopy, an advanced, highly sensitive and reliable method that has been frequently used in many laboratories. From the cell biology point of view, para-cellular barrier function depends on the VE-cadherin/ catenin complex, which closely interacts with actin filaments. Since VE-cadherin provides the backbone of adherence-type junctions in endothelial cells, Bravi et al. (2014) review this molecule in "VE-cadherin at a glance". Furthermore, as has become evident, VE-cadherin and $\mathrm{N}$-cadherin are expressed in certain tumor cells and modulate tumor progression and metastasis, probably by certain cadherin-mediated cell signaling mechanisms. These features might give further insight into the regulatory capacity of endothelial cadherins, a topic addressed by Breier et al. (2014) in "Endothelial cadherins in cancer". The dynamics of VE-cadherin and its interaction with actin filaments under resting and shear stress conditions is summarized by Schnittler et al. (2014) in "Actin dynamics and endothelial cell junctions". The impact of mechanical forces, which are related to actin dynamics in endothelial barrier function, is reviewed by Oldenburg and de Rooij (2014) in "Mechanical control of the endothelial barrier". Fels et al. (2014) address mechanical features of the endothelium in their contribution "Nanomechanics of vascular endothelium". Changes in barrier function are accompanied by dynamic repositioning of actin filaments and VE-cadherins in the cell, an event that requires the essential control of Rho GTPases. This topic is addressed by Amado-Azevedo et al. (2014) within the article "Regulation of the endothelial barrier function: a filum granum of cellular forces, RhoGTPase signaling and microenvironment'.

Opening/activation and resealing of endothelial barriers requires certain post-translational modifications of participating junction proteins. These processes greatly rely on the cellular phosphorylation/dephosphorylation machinery, a topic reviewed by Küppers et al. (2014) in "Phosphatases and 
kinases as regulators of the endothelial barrier function". Stabilization of endothelial barrier function is another important issue that is facilitated by intracellular cAMP and this is focused upon in the article "cAMP with other signaling cues converges on Racl to stabilize the endothelial barrier - a signaling pathway compromised in inflammation", presented by Schlegel and Waschke (2014). Glucocorticoids also mediate the stabilization of barrier function; here, Salvador et al. (2014) address this topic in "Glucocorticoids and endothelial cell barrier function". A further important molecule that is involved in controlling endothelial integrity is the platelet endothelial cell adhesion molecule (PECAM) on which Privratsky and Newman (2014) will concentrate in "PECAM-1: regulator of endothelial junctional integrity".

Under pathological conditions, including trauma and infections, endothelial barrier function is often compromised, being accompanied by loss of integrity and major changes in cell signaling. The concept that hemorrhagic-fever-inducing viruses can directly or indirectly target the endothelium and thereby disorganize diverse biological functions is reviewed by Anon Srikiatkhachorn (2014) in "Vascular events in viral hemorrhagic fevers". Furthermore, infections, traumata and vascular disease conditions are associated with the release of extracellular DNA and RNA into the blood stream or the tissue. This novel idea is taken up by Fischer et al. (2014) in their review entitled "Impact of extracellular RNA on endothelial barrier function". A major event in inflammatory situations is the recruitment to and transmigration of leukocytes through the endothelium into the inflamed tissue; this is of substantial importance in innate immunity and beyond. Leick et al. (2014) summarize these aspects in "Leukocyte recruitment in inflammation: basic concepts and new mechanistic insights based on new models and microscopic imaging technologies".

Organ-specific features of the endothelial barrier in the lung are highlighted in the next two articles by MüllerRedetzky et al. (2014) who focus on "Dynamics of pulmonary endothelial barrier function in acute inflammation: mechanisms and therapeutic perspectives" and Duluc and WojciakStothard (2014) who address "Rho GTPases in the regulation of pulmonary vascular barrier". Indeed, the break-down of the endothelial cell barrier in the lung is one of the most critical problems associated with shock syndrome, a condition that still represents a challenge for appropriate treatment. The other organ-specific articles presented here address issues of the blood-brain-barrier, the tightest endothelial barrier within the body. Engelhardt and Liebner (2014) summarize this topic in "Novel insights into the development and maintenance of the blood-brain barrier". Moreover, tightly linked endothelial cells depend on adhesive interactions that are under the dynamic control of junction-associated-molecules as further outlined by Garrido-Urbani et al. (2014) in "Tight junction dynamics: the role of junctional adhesion molecules (JAMs)". Finally, the delivery of drugs across various types of tightly formed endothelia is not trivial and therefore requires special methodological approaches and drug development. This topic is considered by Rempe et al. (2014) in "Strategies to overcome the barrier: use of nanoparticles as carriers and modulators of barrier properties".

We hope that the readers will find this collection of theme-focused articles not only enjoyable and helpful but also a source of ideas worthy of inclusion in their own work.

Acknowledgments I am grateful to Klaus Preissner, Gießen for constructive discussion. This work was supported by grants of the German Research Council, DFG INST 2105/24-1 and SCHN 430/6-1 to HS.

\section{References}

Amado-Azevedo J, Valent ET, Van Nieuw Amerongen GP (2014) Regulation of the endothelial barrier function: a filum granum of cellular forces, Rho GTPase signaling and microenvironment. Cell Tissue Res. doi:10.1007/s00441-014-1828-6

Bravi L, Dejana E, Lampugnani (2014) MGVE-cadherin at a glance. Cell Tissue Res. doi:10.1007/s00441-014-1843-7

Breier G, Grosser M, Rezaei M (2014) Endothelial cadherins in cancer. Cell Tissue Res. doi:10.1007/s00441-014-1851-7

Duluc L, Wojciak-Stothard B (2014) Rho GTPases in the regulation of pulmonary vascular barrier function. Cell Tissue Res. doi:10.1007/ s00441-014-1805-0

Engelhardt B, Liebner S (2014) Novel insights into the development and maintenance of the blood-brain barrier. Cell Tissue Res. doi:10. 1007/s00441-014-1811-2

Fels J, Jeggle P, Liashkovich I, Peters W, Oberleithner H (2014) Nanomechanics of vascular endothelium. Cell Tissue Res. doi:10. 1007/s00441-014-1853-5

Fischer S, Cabrera-Fuentes HA, Noll T, Preissner KT (2014) Impact of Extracellular RNA on Endothelial Barrier Function. Cell Tissue Res. doi:10.1007/s00441-014-1850-8

Garrido-Urbani S, Bradfield PF, Imhof BA (2014) Tight junction dynamics: the role of junctional adhesion molecules (JAMs). Cell Tissue Res. doi:10.1007/s00441-014-1820-1

Küppers V, Vockel M, Nottebaum AF, Vestweber D (2014) Phosphatases and kinases as regulators of the endothelial barrier function. Cell Tissue Res. doi:10.1007/s00441-014-1812-1

Leick M, Azcutia V, Newton G, Luscinskas FW (2014) Leukocyte recruitment in inflammation: basic concepts and new mechanistic insights based on new models and microscopic imaging technologies. Cell Tissue Res. doi:10.1007/ s00441-014-1809-9

Müller-Redetzky HC, Suttorp N, Witzenrath M (2014) Dynamics of pulmonary endothelial barrier function in acute inflammation: mechanisms and therapeutic perspectives. Cell Tissue Res. doi:10. 1007/s00441-014-1821-0

Oldenburg J, de Rooij J (2014) Mechanical control of the endothelial barrier. Cell Tissue Res. doi:10.1007/s00441-013-1792-6

Privratsky JR, Newman PJ (2014) PECAM-1: regulator of endothelial junctional integrity. Cell Tissue Res. doi:10.1007/ s00441-013-1779-3

Rempe R, Cramer S, Qiao R, Galla H-J (2014) Strategies to overcome the barrier: use of nanoparticles as carriers and modulators of barrier properties. Cell Tissue Res. doi:10. 1007/s00441-014-1819-7 
Salvador E, Shityakov S, Förster C (2014) Glucocorticoids and endothelial cell barrier function. Cell Tissue Res. doi:10.1007/s00441-013-1762-Z

Schlegel N, Waschke J (2014) cAMP with other signaling cues converges on Rac1 to stabilize the endothelial barrier- a signaling pathway compromised in inflammation. Cell Tissue Res. doi:10.1007/ s00441-013-1755-y

Schnittler H, Taha M, Odenthal-Schnittler M, Abu Taha A, Lindemann N, Seebach J (2014) Actin filament dynamics and endothelial junctions. The Ying and Yang between stabilisation and motion. Cell Tissue Res. doi:10.1007/s00441-014-1856-2

Srikiatkhachorn A (2014) Vascular events in viral hemorrhagic fevers. Cell Tissue Res. doi:10.1007/s00441-014-1841-9

Wegener J, Seebach J (2014) Experimental tools to monitor the dynamics of endothelial barrier function: a survey of in vitro approaches. Cell Tissue Res. doi:10.1007/s00441014-1810-3 